Supporting Information: Efficient Synthesis of Highly Functionalized Cyclic Aminimides; Moon, Han, and Kim May 31, 2005.

\title{
Efficient Synthesis of Highly Functionalized Cyclic Aminimides
}

\author{
Bongjin Moon, ${ }^{*}$ Sangbae Han, and Dohyung Kim \\ Department of Chemistry, Sogang University, Seoul 121-742, Korea
}

\section{Supporting Information}

Experimental procedures and characterization data for the preparation of compounds

\section{2-22 and 23-29.}

${ }^{1} \mathrm{H}-\mathrm{NMR}$ and ${ }^{13} \mathrm{C}-\mathrm{NMR}$ spectra were obtained using a Varian Gemini-300 (300 $\mathrm{MHz}$ for ${ }^{1} \mathrm{H}$, and $75 \mathrm{MHz}$ for ${ }^{13} \mathrm{C}$ ), or a Varian Inova-500 (500 MHz for ${ }^{1} \mathrm{H}$, and 125 $\mathrm{MHz}$ for ${ }^{13} \mathrm{C}$ ) spectrometer. Chemical shifts are reported relative to tetramethylsilane peak $(\delta 0.00)$ or solvent peak ( $\delta 7.26$ for $\mathrm{CDCl}_{3}$ in ${ }^{1} \mathrm{H} \mathrm{NMR}, \delta 77.2$ for $\mathrm{CDCl}_{3}$ in ${ }^{13} \mathrm{C}$ NMR). Elemental analyses were performed by the Organic Chemistry Research Center at Sogang University using a Carlo Erba EA 1180 elemental analyzer. Electron spray ionization (ESI) low resolution mass spectra were obtained with a Waters Micromass ZQ mass spectrometer. IR spectra were obtained using a Thermo-Nicolet Avartar-320 IR spectrometer with a single bounce ATR accessory (Ge crystal). THF and ethyl ether were distilled from sodium benzophenone ketyl. Methylene chloride was distilled from calcium hydride. $N, N$-dimethylformamide (DMF) was dried over $4 \AA$ molecular sieves. $N$, $N$-dimethylhydrazine, $\quad \mathrm{N}$-aminomorpholine, $\quad \mathrm{N}$-aminopiperidine, $\quad 1$-amino-4methylpiperazine were purchased from Aldrich. All other chemicals were used as received otherwise specified. 
Supporting Information: Efficient Synthesis of Highly Functionalized Cyclic Aminimides; Moon, Han, and Kim May 31, 2005.

\section{General Procedure:}

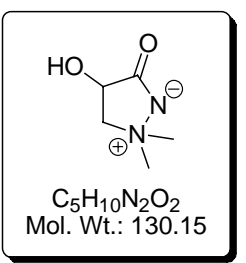

4-Hydroxy-1,1-dimethyl-pyrazolidin-3-one (12): A solution of epoxy methyl ester 8 (1.9 g, $18.6 \mathrm{mmol})$, dimethyl hydrazine (A) $(1.70 \mathrm{~mL}, 22.4 \mathrm{mmol})$ in $i$-PrOH (5 $\mathrm{mL}$ ) was stirred for $3 \mathrm{~h}$ at room temperature. The resulting white precipitates were filtered through a filter paper, washed with diethyl ether, and dried under vacuum to give the product as a white solid (2.3 g, 95\%): m.p.: $266 \square ;{ }^{1} \mathrm{H} \mathrm{NMR}\left(500 \mathrm{MHz}, \mathrm{D}_{2} \mathrm{O}\right) \delta$ $4.67(\mathrm{dd}, J=7.5 \mathrm{~Hz}$ and $5.5 \mathrm{~Hz}, 1 \mathrm{H}), 4.03(\mathrm{dd}, J=7.5 \mathrm{~Hz}$ and $12.3 \mathrm{~Hz}, 1 \mathrm{H}), 3.64$ (dd, $J$ $=5.5 \mathrm{~Hz}$ and $12.3 \mathrm{~Hz}, 1 \mathrm{H}), 3.18(\mathrm{~s}, 3 \mathrm{H}), 3.11(\mathrm{~s}, 3 \mathrm{H}) ;{ }^{13} \mathrm{C} \mathrm{NMR}\left(125 \mathrm{MHz}, \mathrm{D}_{2} \mathrm{O}\right)$ 179.85, 71.44, 69.93, 58.42, 57.38; IR (Ge ATR) 3085, 3027, 1606, 1342, 1305, 1259, $1112 \mathrm{~cm}^{-1}$. Anal. Calcd for $\mathrm{C}_{5} \mathrm{H}_{10} \mathrm{~N}_{2} \mathrm{O}_{2}$ : C, 46.14; H, 7.74; N, 21.52. Found: C, 46.22; H, $7.36 ; \mathrm{N}, 21.30$.

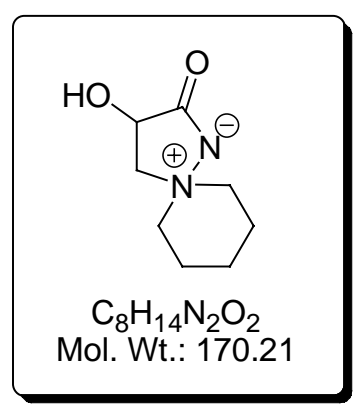

3-Hydroxy-1,5-diaza-spiro[4.5]decan-2-one (13): Yield = 71\%; m.p: $263{ }^{\circ} \mathrm{C}$; ${ }^{1} \mathrm{H}$ NMR $\left(500 \mathrm{MHz}, \mathrm{D}_{2} \mathrm{O}\right) \delta 4.62(\mathrm{dd}, J=5.0$ and $7.5 \mathrm{~Hz}, 1 \mathrm{H}), 4.00(\mathrm{dd}, J=7.5$ and $12.5 \mathrm{~Hz}, 1 \mathrm{H}), 3.59$ (dd, $J=5.0$ and $12.5 \mathrm{~Hz}, 1 \mathrm{H}), 3.4 \sim 3.2(\mathrm{~m}, 4 \mathrm{H}), 1.88(\mathrm{~m}, 2 \mathrm{H}), 1.74$ (m, 2H), 1.59 (m, 1H), 1.50 (m, 1H); ${ }^{13} \mathrm{C}$ NMR (125 MHz, $\left.\mathrm{D}_{2} \mathrm{O}\right)$ 179.80, 70.19, 67.39, 66.30, 21.68, 21.50, 20.48; IR (Ge ATR) 2945, 1565, 1454, 1388, 1316, 1106, $1050 \mathrm{~cm}^{-}$ ${ }^{1}$. Anal. Calcd for $\mathrm{C}_{8} \mathrm{H}_{14} \mathrm{~N}_{2} \mathrm{O}_{2}$ : C, 56.45; H, 8.29; N, 16.46. Found: C, 56.49; H, 8.50; N, 16.30 . 
Supporting Information: Efficient Synthesis of Highly Functionalized Cyclic Aminimides; Moon, Han, and Kim May 31, 2005.

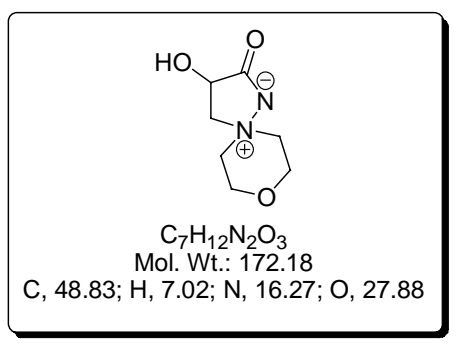

3-Hydroxy-8-oxa-1,5-diazaspiro[4,5]decane-2-one (14): Yield = 49\%; m.p.: $252 \square ;{ }^{1} \mathrm{H}$ NMR $\left(500 \mathrm{MHz}, \mathrm{D}_{2} \mathrm{O}\right) \delta 4.65(\mathrm{dd}, J=5.5$ and $7.5 \mathrm{~Hz}, 1 \mathrm{H}), 4.09(\mathrm{dd}, J=7.5$ and $12.5 \mathrm{~Hz}, 1 \mathrm{H}), 4.08(\mathrm{~m}, 2 \mathrm{H}), 3.91(\mathrm{ddd}, J=5.0,5.0$, and $5.0 \mathrm{~Hz}, 1 \mathrm{H}), 3.88(\mathrm{ddd}, J=$ $5.0 \mathrm{~Hz}, 5.0$, and $5.0 \mathrm{~Hz}, 1 \mathrm{H}), 3.73(\mathrm{dd}, J=5.5$ and $12.5 \mathrm{~Hz}, 1 \mathrm{H}), 3.59$ (ddd, $J=3.0,7.5$ and $12.0 \mathrm{~Hz}, 1 \mathrm{H}), 3.53(\mathrm{ddd}, J=3.0,8.0$ and $12.5 \mathrm{~Hz}, 1 \mathrm{H}), 3.43(\mathrm{~m}, 1 \mathrm{H}), 3.32(\mathrm{~m}, 1 \mathrm{H})$; ${ }^{13} \mathrm{C}$ NMR (125 MHz, D $\left.2 \mathrm{O}\right)$ 180.32, 70.07, 68.70 (br), 65.71, 64.58, 62.40, 62.28; IR (Ge ATR) $3079,2875,1593,1467,1392,1292,1132,1060 \mathrm{~cm}^{-1}$. Anal. Calcd for $\mathrm{C}_{7} \mathrm{H}_{12} \mathrm{~N}_{2} \mathrm{O}_{3}$ : C, 48.83; H, 7.02; N, 16.27. Found: C, 48.53; H, 7.52; N, 16.13. LRMS (ESI): $m / z, 194.7$ (195.1 calcd for $\mathrm{C}_{7} \mathrm{H}_{12} \mathrm{~N}_{2} \mathrm{O}_{3} \mathrm{Na}[\mathrm{M}+\mathrm{Na}]^{+}$), 172.7 (173.1 calcd for $\left.\mathrm{C}_{7} \mathrm{H}_{13} \mathrm{~N}_{2} \mathrm{O}_{3}[\mathrm{M}+\mathrm{H}]^{+}\right)$.

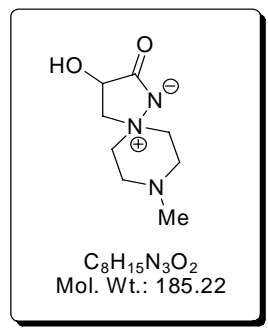

3-Hydroxy-8-methyl-1,5,8-triaza-spiro[4.5]decan-2-one (15): yield $=45 \%$; m.p: $211 \square ;{ }^{1} \mathrm{H}$ NMR (500 MHz, $\left.\mathrm{D}_{2} \mathrm{O}\right) \delta 4.63(\mathrm{dd}, J=5.3$ and $7.5 \mathrm{~Hz}, 1 \mathrm{H}), 4.05(\mathrm{dd}, J$ $=7.5$ and $12.5 \mathrm{~Hz}, 1 \mathrm{H}$ ), 3.68 (dd, $J=5.3$ and $12.5 \mathrm{~Hz}, 1 \mathrm{H}), 3.50$ (br m, 1H), 3.44 (br m, 1H), 3.42 (br m, 1H), 3.34 (br m, 1H), 2.86 (br m, 2H), 2.72 (br m, 2H), 2.26 (s, 3H); ${ }^{13} \mathrm{C}$ NMR (125 MHz, $\left.\mathrm{D}_{2} \mathrm{O}\right)$ 180.17, 70.07, 65.59, 64.48, 49.39, 49.26, 43.99, 23.89; IR (Ge ATR) 3443, 2869, 1652, 1570, 1468, 1447, 1370, 1291, 1146, $1095 \mathrm{~cm}^{-1}$. LRMS (ESI): $m / z 207.8\left(208.1\right.$ calcd for $\left.\mathrm{C}_{8} \mathrm{H}_{15} \mathrm{~N}_{3} \mathrm{O}_{2} \mathrm{Na}[\mathrm{M}+\mathrm{Na}]^{+}\right), 185.8$ (186.1 calcd for $\left.\mathrm{C}_{8} \mathrm{H}_{16} \mathrm{~N}_{3} \mathrm{O}_{2}[\mathrm{M}+\mathrm{H}]^{+}\right)$. 
Supporting Information: Efficient Synthesis of Highly Functionalized Cyclic Aminimides; Moon, Han, and Kim May 31, 2005.

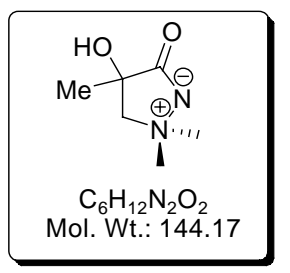

4-Hydroxy-1,1,4-trimethyl-pyrazolidin-3-one (16): A solution of 2-methyloxirane-2-carboxylic acid methyl ester $(9,300 \mathrm{mg}, 2.60 \mathrm{mmol})$, dimethyl hydrazine $(236 \mu \mathrm{L}, 3.10 \mathrm{mmol})$ in $i$-PrOH $(3 \mathrm{~mL})$ was stirred at room temperature for $6 \mathrm{~h}$. The resulting precipitates were collected by filtration, washed with diethyl ether, and dried under vacuum to give cyclic aminimide 16 as a white solid (251 mg, 67\%): m.p: $252 \square$; ${ }^{1} \mathrm{H}$ NMR (500 MHz, D $\left.\mathrm{D}_{2} \mathrm{O}\right) \delta 3.93(\mathrm{~d}, J=13.0 \mathrm{~Hz}, 1 \mathrm{H}), 3.76(\mathrm{~d}, J=13.0 \mathrm{~Hz}, 1 \mathrm{H}), 3.23(\mathrm{~s}$, $3 \mathrm{H}), 3.15$ (s, 3H), 1.37 (s, 3H); ${ }^{13} \mathrm{C}$ NMR (125 MHz, $\left.\mathrm{D}_{2} \mathrm{O}\right)$ 180.89, 77.96, 74.56, 58.28, 57.73, 22.40; IR (film) 3065, 1620, 1605, 1472, 1388, 1208, 1175, 1147, $999 \mathrm{~cm}^{-1}$. Anal. Calcd for $\mathrm{C}_{6} \mathrm{H}_{12} \mathrm{~N}_{2} \mathrm{O}_{2}$ : C, 49.98; H, 8.39; N, 19.43. Found: C, 49.80; H, 8.57; N, 19.58 .

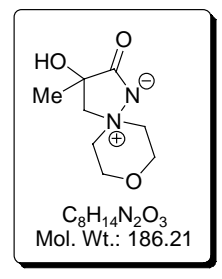

3-Hydroxy-3-methyl-8-oxa-1,5-diaza-spiro[4.5]decan-2-one (17): Yield = 53\%; m.p: $277 \square ;{ }^{1} \mathrm{H}$ NMR (500 MHz, $\left.\mathrm{D}_{2} \mathrm{O}\right) \delta 4.08(\mathrm{~m}, 2 \mathrm{H}), 4.03(\mathrm{~d}, J=13.0 \mathrm{~Hz}, 1 \mathrm{H})$, $3.88(\mathrm{~m}, 2 \mathrm{H}), 3.75(\mathrm{~d}, J=13.0 \mathrm{~Hz}, 1 \mathrm{H}), 3.61(\mathrm{ddd}, J=3.0,7.5$, and $13.0 \mathrm{~Hz}, 1 \mathrm{H}), 3.51$ $(\mathrm{m}, 2 \mathrm{H}), 3.33(\mathrm{~m}, 1 \mathrm{H}), 1.38(\mathrm{~s}, 3 \mathrm{H}) ;{ }^{13} \mathrm{C} \mathrm{NMR}\left(125 \mathrm{MHz}, \mathrm{D}_{2} \mathrm{O}\right) 181.39,76.40,73.27$ (br), 65.57, 64.73, 62.38, 62.34, 22.41; IR (Ge ATR) 3102, 1588, 1476, 1408, 1389, 1294, 1166, 1127, $1058 \mathrm{~cm}^{-1}$. LRMS (ESI): $m / z, 208.7$ (209.1 calcd for $\mathrm{C}_{8} \mathrm{H}_{14} \mathrm{~N}_{2} \mathrm{O}_{3} \mathrm{Na}$ $\left.[\mathrm{M}+\mathrm{Na}]^{+}\right), 186.7\left(187.1\right.$ calcd for $\left.\mathrm{C}_{8} \mathrm{H}_{15} \mathrm{~N}_{2} \mathrm{O}_{3}[\mathrm{M}+\mathrm{H}]^{+}\right)$.

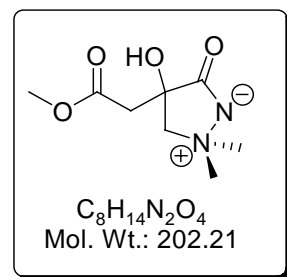

(4-Hydroxy-1,1-dimethyl-3-oxo-pyrazolidin-4-yl)-acetic acid methyl ester (18): Yield = 62\%; m.p: $210 \square:{ }^{1} \mathrm{H}$ NMR $\left(500 \mathrm{MHz}, \mathrm{D}_{2} \mathrm{O}\right) \delta 4.17(\mathrm{~d}, J=22.0 \mathrm{~Hz}, 1 \mathrm{H})$, 4.10 (d, $J=22.0 \mathrm{~Hz}, 1 \mathrm{H}), 3.67$ (s, 3H), 3.33 (s, 3H), 3.26 (s, 3H), 2.99 (d, J = 28.5 Hz, 
Supporting Information: Efficient Synthesis of Highly Functionalized Cyclic Aminimides; Moon, Han, and Kim May 31, 2005.

$1 \mathrm{H}), 2.84(\mathrm{~d}, J=28.5 \mathrm{~Hz}, 1 \mathrm{H}) ;{ }^{13} \mathrm{C}$ NMR $\left(125 \mathrm{MHz}, \mathrm{D}_{2} \mathrm{O}\right) 178.95,172.35,78.32,73.27$, 58.45, 57.72, 52.62, 40.10; IR (Ge ATR) 3032, 1730, 1622, 1349, 1223, $1202 \mathrm{~cm}^{-1}$. Anal. Calcd for $\mathrm{C}_{8} \mathrm{H}_{14} \mathrm{~N}_{2} \mathrm{O}_{4}$ : C, 47.52; H, 6.98; N, 13.85. Found: C, 47.60; H, 7.11; N, 13.62.

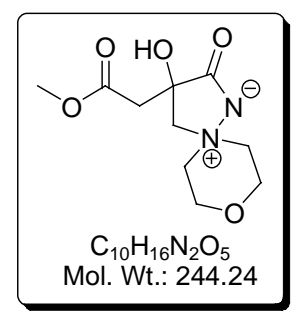

(3-Hydroxy-2-oxo-8-oxa-1,5-diaza-spiro[4.5]dec-3-yl)-acetic acid methyl ester (19): Yield $=35 \%$; m.p: $212 \square ;{ }^{1} \mathrm{H}$ NMR $\left(500 \mathrm{MHz}, \mathrm{D}_{2} \mathrm{O}\right) \delta 4.18(\mathrm{~d}, J=13.0 \mathrm{~Hz}$, 1H), 4.09 (m, 2H), 4.01 (d, $J=13.0 \mathrm{~Hz}, 1 \mathrm{H}), 3.90$ (br d, $J=13.5 \mathrm{~Hz}, 2 \mathrm{H}), 3.64$ (m, 1H), 3.63 (s, 3H), 3.56 (m, 2H), 3.35 (br d, $J=12.5 \mathrm{~Hz}, 1 \mathrm{H}$ ), 2.93 (d, $J=17.0 \mathrm{~Hz}, 1 \mathrm{H}), 2.77$ $(\mathrm{d}, J=17.0 \mathrm{~Hz}, 1 \mathrm{H}) ;{ }^{13} \mathrm{C} \mathrm{NMR}\left(125 \mathrm{MHz}, \mathrm{D}_{2} \mathrm{O}\right)$ 179.39, 172.32, 76.73, 72.14 (br), 65.83, 64.73, 62.44, 62.36, 52.63, 40.06; IR (KBr) 3427, 3135, 2958, 2928, 2903, 1725 , 1604, 1448, 1431, 1356, 1225, 1203, 1127, $1036 \mathrm{~cm}^{-1}$. Anal. Calcd for $\mathrm{C}_{10} \mathrm{H}_{16} \mathrm{~N}_{2} \mathrm{O}_{5}: \mathrm{C}$, 49.17; H, 6.60; N, 11.47. Found: C, 49.20; H, 6.75; N, 11.59 .

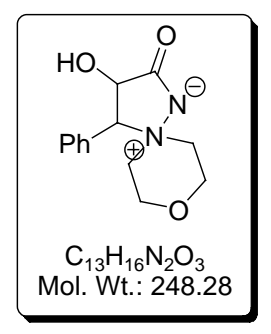

3-Hydroxy-4-phenyl-8-oxa-1,5-diaza-spiro[4.5]decan-2-one (20): Yield = 75\%; m.p: $199 \square:{ }^{1} \mathrm{H}$ NMR (500 MHz, $\left.\mathrm{D}_{2} \mathrm{O}\right) \delta$ 7.59-7.49 (m, 5H), $5.18(\mathrm{~d}, J=9.3 \mathrm{~Hz}$, $1 \mathrm{H}), 4.76(\mathrm{~d}, J=9.3 \mathrm{~Hz}, 1 \mathrm{H}), 4.18(\mathrm{ddd}, J=13.0,11.5$ and $1.5 \mathrm{~Hz}, 1 \mathrm{H}), 4.02$ (ddd, $J=$ $2.0,10.5$ and $12.5 \mathrm{~Hz}, 1 \mathrm{H}), 3.86(\mathrm{dd}, J=3.5,13.0 \mathrm{~Hz}, 1 \mathrm{H}), 3.70(\mathrm{br} \mathrm{d}, J=\sim 11 \mathrm{~Hz}, 1 \mathrm{H})$, $3.67(\mathrm{dt}, J=4$ and $12.5 \mathrm{~Hz}, 1 \mathrm{H}), 3.32(\mathrm{~d}, J=13.0 \mathrm{~Hz}, 1 \mathrm{H}), 3.01(\mathrm{dd}, J=1.5$ and 12.0 $\mathrm{Hz}, 1 \mathrm{H}), 2.95$ (ddd, $J=4.0,12.5$ and $12.5 \mathrm{~Hz}, 1 \mathrm{H}) ;{ }^{13} \mathrm{C}$ NMR $\left(125 \mathrm{MHz}, \mathrm{D}_{2} \mathrm{O}\right) 180.36$, $131.77,131.34,129.87,127.27,84.84,71.52$, 62.36, 62.18, 61.48, 59.83; IR (KBr) 3422, 3096, 2954, 2929, 2891, 1590, 1472, 1459, 1446, 1402, 1392, 1289, 1125, 1072, 937 $\mathrm{cm}^{-1}$. Anal. Calcd for $\mathrm{C}_{13} \mathrm{H}_{16} \mathrm{~N}_{2} \mathrm{O}_{3}: \mathrm{C}, 62.89 ; \mathrm{H}, 6.50 ; \mathrm{N}, 11.28$. Found: C, 62.92; $\mathrm{H}$, 6.62; N, 11.13 . 
Supporting Information: Efficient Synthesis of Highly Functionalized Cyclic Aminimides; Moon, Han, and Kim May 31, 2005.

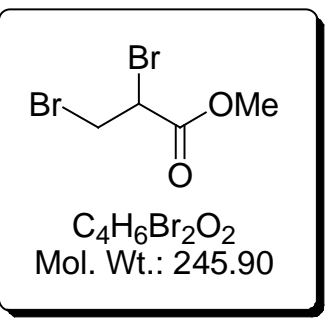

2,3-Dibromo-propionic acid methyl ester: To a solution of methyl acrylate $(10.0 \mathrm{~g}, 0.12 \mathrm{mmol})$ in $\mathrm{CH}_{2} \mathrm{Cl}_{2}(30 \mathrm{~mL})$ was slowly added bromine $(5.95 \mathrm{~mL}, 0.12$ mmol) using a dropping funnel at $0{ }^{\circ} \mathrm{C}$. The resulting solution was allowed to stir at 0 ${ }^{\circ} \mathrm{C}$ for $1 \mathrm{~h}$. The reaction was quenched by adding a saturated sodium thiosulfate solution $(20 \mathrm{~mL})$. The resulting suspension was allowed to stir for $1 \mathrm{~h}$ at room temperature. The mixture was diluted with $\mathrm{CH}_{2} \mathrm{Cl}_{2}(10 \mathrm{~mL})$ and extracted with $\mathrm{CH}_{2} \mathrm{Cl}_{2}$ $(3 \times 10 \mathrm{~mL})$. The organic layers were washed with brine, dried over $\mathrm{Na}_{2} \mathrm{SO}_{4}$, and concentrated. Purification of the crude product by flash chromatography (Hex:EtOAc $=3: 1)$ on silica gel gave the product as a yellow liquid $(27.5 \mathrm{~g}, 95 \%):{ }^{1} \mathrm{H}$ NMR (300 $\left.\mathrm{MHz}, \mathrm{CDCl}_{3}\right) \delta 4.45(\mathrm{dd}, J=4.5$ and $11.4 \mathrm{~Hz}, 1 \mathrm{H}), 3.93(\mathrm{dd}, J=9.9$ and $11.4 \mathrm{~Hz}, 1 \mathrm{H})$, $3.85(\mathrm{~s}, 3 \mathrm{H}), 3.68$ (dd, $J=4.5$ and $9.9 \mathrm{~Hz}, 1 \mathrm{H})$.

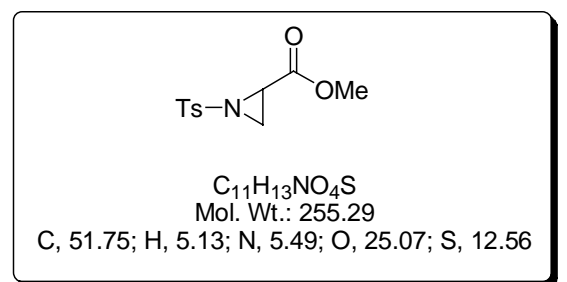

1-(Toluene-4-sulfonyl)-aziridine-2-carboxylic acid methyl ester (21): To a suspension of 2,3-dibromo-propionic acid methyl ester $(2.60 \mathrm{~mL}, 10.8 \mathrm{mmol})$ and $\mathrm{K}_{2} \mathrm{CO}_{3}(4.5 \mathrm{~g}, 32.5 \mathrm{mmol})$ in $\mathrm{CH}_{3} \mathrm{CN}(15 \mathrm{~mL})$ was added toluenesulfonamide (1.85 g, $10.8 \mathrm{mmol}$ ) at rt. Then the reaction mixture was heated at $80{ }^{\circ} \mathrm{C}$ overnight. The reaction was quenched by adding $\mathrm{H}_{2} \mathrm{O}$ and the mixture was extracted with EtOAc $(15 \mathrm{~mL} \times 3)$. The combined organic layers were washed with brine, dried over $\mathrm{Na}_{2} \mathrm{SO}_{4}$, and concentrated. The residue was purified by flash chromatography $(\mathrm{Hex}: \mathrm{EtOAc}=3: 1)$ on silica gel to give the product as a yellow liquid 21 (1.5 g, 56\%): ${ }^{1} \mathrm{H}$ NMR (500 MHz, $\left.\mathrm{CDCl}_{3}\right) \delta 7.83(\mathrm{~d}, J=8.4 \mathrm{~Hz}, 2 \mathrm{H}), 7.35(\mathrm{~d}, J=8.1 \mathrm{~Hz}, 2 \mathrm{H}), 3.72(\mathrm{~s}, 3 \mathrm{H}), 3.33(\mathrm{dd}, J=$ 4.2 and $7.2 \mathrm{~Hz}, 1 \mathrm{H}), 2.75(\mathrm{~d}, J=7.2 \mathrm{~Hz}, 1 \mathrm{H}), 2.55(\mathrm{~d}, J=4.2 \mathrm{~Hz}, 1 \mathrm{H}), 2.44(\mathrm{~s}, 3 \mathrm{H}) ;{ }^{13} \mathrm{C}$ NMR (75 MHz, $\mathrm{CDCl}_{3}$ ) 178.31, 142.89, 136.37, 129.41, 125.27, 52.48, 31.24, 20.90, 
Supporting Information: Efficient Synthesis of Highly Functionalized Cyclic Aminimides; Moon, Han, and Kim May 31, 2005.

18.43 .

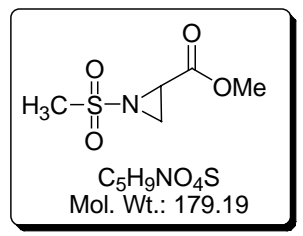

1-Methanesulfonyl-aziridine-2-carboxylic acid methyl ester (22): To a solution of 2,3-dibromo-propionic acid methyl ester (2.20 g, $9.02 \mathrm{mmol}), \mathrm{K}_{2} \mathrm{CO}_{3}(3.7 \mathrm{~g}, 27.1$ mmol) in $\mathrm{CH}_{3} \mathrm{CN}(10 \mathrm{~mL})$ was added dropwise methanesulfonamide $(858 \mathrm{mg}, 9.02$ $\mathrm{mmol})$ at room temperature. Then the reaction mixture was stirred at $80 \square$ overnight. After the starting material disappeared judging from TLC analysis, the reaction was quenched with $\mathrm{H}_{2} \mathrm{O}$, extracted with EtOAc $(15 \mathrm{~mL} \times 3)$. The organic layers were washed with brine, dried over $\mathrm{Na}_{2} \mathrm{SO}_{4}$, and concentrated. Then the residue was purified by flash chromatography (Hexane/EtOAc $=3 / 1)$ on silica gel to give 22 as a yellow liquid (945 mg, 52\%): ${ }^{1} \mathrm{H}$ NMR (300 MHz, $\left.\mathrm{CDCl}_{3}\right) \delta 3.78(\mathrm{~s}, 3 \mathrm{H}), 3.28$ (dd, $J=4.2$ and $7.2 \mathrm{~Hz}, 1 \mathrm{H}), 3.12(\mathrm{~s}, 3 \mathrm{H}), 2.74(\mathrm{dd}, J=2.1$ and $7.2 \mathrm{~Hz}, 1 \mathrm{H}), 2.60(\mathrm{dd}, J=2.1$ and 4.2 $\mathrm{Hz}, 1 \mathrm{H})$;

${ }^{13} \mathrm{C}$ NMR (75 MHz, $\left.\mathrm{CDCl}_{3}\right)$ 179.23, 54.26, 42.11, 36.87, 22.46.

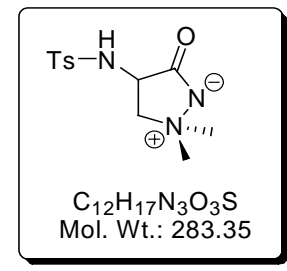

$N$-(5,5-Dimethyl-2-oxo-pyrrolidin-3-yl)-4-methyl-benzenesulfonamide (24): A solution of 1-(toluene-4-sulfonyl)-aziridine-2-carboxylic acid methyl ester (21, 230 $\mathrm{mg}, 0.9 \mathrm{mmol})$ and dimethyl hydrazine $(82 \mu \mathrm{L}, 1.1 \mathrm{mmol})$ in $i$-PrOH $(1 \mathrm{~mL})$ was stirred at $\mathrm{rt}$ for $5 \mathrm{~h}$. The resulting precipitates were filtered, washed with diethyl ether, and dried under vacuum to give the product as a white solid (189 mg, 74\%): m.p: $223 \square{ }^{1} \mathrm{H}$ NMR (500 MHz, $\left.\mathrm{D}_{2} \mathrm{O}\right) \delta 7.67(\mathrm{~d}, J=7.8 \mathrm{~Hz}, 2 \mathrm{H}), 7.35$ (d, $\left.J=7.8 \mathrm{~Hz}, 2 \mathrm{H}\right), 4.46$ (t, $J=$ $8.5 \mathrm{~Hz}, 1 \mathrm{H}), 3.89(\mathrm{dd}, J=7.5$ and $11.5 \mathrm{~Hz}, 1 \mathrm{H}), 3.34(\mathrm{dd}, J=8.0$ and $12.0 \mathrm{~Hz}, 1 \mathrm{H})$, 3.09 (s, 3H), 3.02 (s, 3H), 2.32 (s, 3H); ${ }^{13} \mathrm{C}$ NMR (125 MHz, $\left.\mathrm{D}_{2} \mathrm{O}\right)$ 176.14, 145.73, 135.21, 130.43, 126.99, 68.10, 58.13, 57.16, 55.28, 20.88; IR (Ge ATR) 3036, 2840, 1636, 1598, 1327, 1162, 1094, $919 \mathrm{~cm}^{-1}$. Anal. Calcd for $\mathrm{C}_{12} \mathrm{H}_{17} \mathrm{~N}_{3} \mathrm{O}_{3} \mathrm{~S}: \mathrm{C}, 50.87 ; \mathrm{H}$, 
Supporting Information: Efficient Synthesis of Highly Functionalized Cyclic Aminimides; Moon, Han, and Kim May 31, 2005.

6.05; N, 14.83. Found: C, 50.72; H, 6.14; N, 14.95.

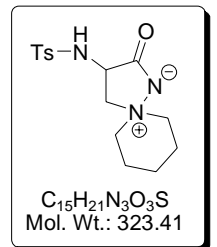

4-Methyl- $N$-(2-oxo-1,5-diaza-spiro[4.5]dec-3-yl)-benzenesulfonamide (25): $\quad \mathrm{A}$ solution of 1-(toluene-4-sulfonyl)-aziridine-2-carboxylic acid methyl ester 21 (370 mg, $1.45 \mathrm{mmol})$ and $N$-aminopiperidine $(188 \mu \mathrm{L}, 1.74 \mathrm{mmol})$ in $i$-PrOH $(2 \mathrm{~mL})$ was stirred at room temperature for $3 \mathrm{~h}$. The resulting precipitates were isolated by filtration, washed with diethyl ether, and dried under vacuum to give $\mathbf{2 5}$ as a white solid (375 $\mathrm{mg}$, 80\%): m.p: $230 \square$; ${ }^{1} \mathrm{H}$ NMR (500 MHz, $\left.\mathrm{D}_{2} \mathrm{O}\right) \delta 7.71(\mathrm{~d}, J=8.5 \mathrm{~Hz}, 2 \mathrm{H}), 7.39$ (d, $J=$ $8.5 \mathrm{~Hz}, 2 \mathrm{H}), 4.39$ (t, $J=8.0 \mathrm{~Hz}, 1 \mathrm{H}), 3.87(\mathrm{dd}, J=8.0$ and $12.0 \mathrm{~Hz}, 1 \mathrm{H}), 3.26(\mathrm{dd}, J=$ 8.0 and $12.5 \mathrm{~Hz}, 1 \mathrm{H}), 3.23(\mathrm{~m}, 2 \mathrm{H}), 3.18(\mathrm{~m}, 2 \mathrm{H}), 2.35(\mathrm{~s}, 3 \mathrm{H}), 1.83(\mathrm{~m}, 2 \mathrm{H}), 1.60(\mathrm{~m}$, 2H), 1.48 (m, 2H); ${ }^{13} \mathrm{C}$ NMR (125 MHz, $\left.\mathrm{D}_{2} \mathrm{O}\right)$ 176.05, 145.76, 135.35, 130.45, 127.04, 67.36, 65.95, 64.65, 54.21, 21.60, 21.32, 20.89, 20.36; IR (Ge ATR) 3035, 2946, 1616, 1433, 1315, 1159, $1096 \mathrm{~cm}^{-1}$. Anal. Calcd for $\mathrm{C}_{15} \mathrm{H}_{21} \mathrm{~N}_{3} \mathrm{O}_{3} \mathrm{~S}: \mathrm{C}, 55.71 ; \mathrm{H}, 6.54 ; \mathrm{N}, 12.99$. Found: C, 55.67; H, 6.51; N, 12.99 .

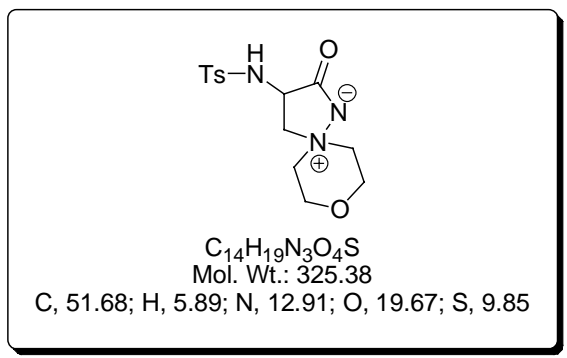

4-Methyl- $N$-(2-oxo-8-oxa-1,5-diaza-spiro[4,5]dec-3-yl)-benzenesulfoamide

(26): A solution of 1-(toluene-4-sulfonyl)-aziridine-2-carboxylic acid methyl ester 21 (226 mg, $0.89 \mathrm{mmol})$ and $N$-aminomorpholine $(102.6 \mu \mathrm{L}, 1.06 \mathrm{mmol})$ in $i$-PrOH $(1 \mathrm{~mL})$ was stirred at $\mathrm{rt}$ for $6 \mathrm{~h}$. The resulting precipitates were filtered, washed with diethyl ether, and dried under vacuum to give the product as a white solid (223 mg, 77\%): m.p: $236 \square ;{ }^{1} \mathrm{H}$ NMR (300 MHz, $\left.\mathrm{D}_{2} \mathrm{O}\right) \delta 7.84(\mathrm{~d}, J=8.4 \mathrm{~Hz}, 2 \mathrm{H}), 7.52(\mathrm{~d}, J=7.8 \mathrm{~Hz}, 2 \mathrm{H})$, $4.55(\mathrm{t}, J=8.1 \mathrm{~Hz}, 1 \mathrm{H}), 4.11(\mathrm{~m}, 3 \mathrm{H}), 3.94(\mathrm{~m}, 2 \mathrm{H}), 3.57$ (m, 3H), 3.39 (m, 2H), 2.48 (s, $3 \mathrm{H}) ;{ }^{13} \mathrm{C}$ NMR (125 MHz, $\left.\mathrm{D}_{2} \mathrm{O}\right)$ 184.49, 145.80, 130.45, 129.11, 127.05, 95.01, 65.31, 64.61, 62.20, 54.01, 23.88, 20.89; IR (film) 3068, 2950, 1628, 1415, 1322, $1163 \mathrm{~cm}^{-1}$. Anal. Calcd for $\mathrm{C}_{14} \mathrm{H}_{19} \mathrm{~N}_{3} \mathrm{O}_{4} \mathrm{~S}$ : C, 51.68; H, 5.89; N, 12.91; S, 9.85. Found: C, 51.56; H, 
Supporting Information: Efficient Synthesis of Highly Functionalized Cyclic Aminimides; Moon, Han, and Kim May 31, 2005.

$6.26 ; \mathrm{N}, 12.88 ; \mathrm{S}, 10.06$.

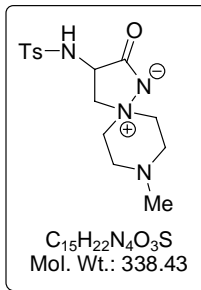

4-Methyl-N-(8-methyl-2-oxo-1,5,8-triaza-spiro[4.5]dec-3-yl)-

benzenesulfonamide (27): A solution of 1-(toluene-4-sulfonyl)-aziridine-2-carboxylic acid methyl ester 21 (415 mg, $1.63 \mathrm{mmol}$ ), 1-amino-4-methylpiperazine (235 $\mu \mathrm{L}, 1.96$ $\mathrm{mmol})$ in $i$-PrOH $(2 \mathrm{~mL})$ was stirred at room temperature for $4 \mathrm{~h}$. The resulting precipitates were isolated by filtration, washed with diethyl ether, and dried under vacuum to give cyclic aminimide 27 as a white solid (458 mg, 83\%): m.p: $211 \square ;{ }^{1} \mathrm{H}$ NMR (500 MHz, D $2 \mathrm{O}) \delta 7.70(\mathrm{~d}, J=8.3 \mathrm{~Hz}, 2 \mathrm{H}), 7.38(\mathrm{~d}, J=8.3 \mathrm{~Hz}, 2 \mathrm{H}), 4.40(\mathrm{t}, J=$ $8.0 \mathrm{~Hz}, 1 \mathrm{H}), 3.92(\mathrm{~m}, 1 \mathrm{H}), 3.38(\mathrm{dd}, J=7.5$ and $12.0 \mathrm{~Hz}, 1 \mathrm{H}), 3.29(\mathrm{~m}, 4 \mathrm{H}), 2.81(\mathrm{~m}$, 2H), 2.65 (m, 2H), 2.34 (s, 3H), 2.23 (s, 3H); ${ }^{13} \mathrm{C}$ NMR (125 MHz, $\left.\mathrm{D}_{2} \mathrm{O}\right)$ 176.34, 145.77, $135.27,130.46,130.10,127.05,125.97,65.26,64.46,54.06,49.18,43.96,23.89,20.89$;

IR (film) 3256, 3040, 2845, 2750, 1593, 1439, 1332, 1116, $1096 \mathrm{~cm}^{-1}$. Anal. Calcd for $\mathrm{C}_{15} \mathrm{H}_{22} \mathrm{~N}_{4} \mathrm{O}_{3} \mathrm{~S}$ : C, 53.23; H, 6.55. Found: C, 53.16; H, 6.79. LRMS (ESI): $\mathrm{m} / z$ (361.1 calcd for $\mathrm{C}_{15} \mathrm{H}_{22} \mathrm{~N}_{4} \mathrm{O}_{3} \mathrm{SNa}[\mathrm{M}+\mathrm{Na}]^{+}$), 339.0 (339.14 calcd for $\mathrm{C}_{15} \mathrm{H}_{23} \mathrm{~N}_{4} \mathrm{O}_{3} \mathrm{~S}$ $\left.[\mathrm{M}+\mathrm{H}]^{+}\right)$.

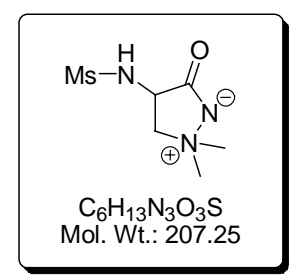

$N$-(1,1-Dimethyl-3-oxo-pyrrolidin-4-yl)-methanesulfonamide $\quad(28): \quad \mathrm{A}$ solution of 1-methanesulfonylaziridine-2-carboxylic acid methyl ester 22 (245 mg, 1.36 $\mathrm{mmol})$ and dimethyl hydrazine $(125 \mu \mathrm{L}, 1.64 \mathrm{mmol})$ in $i$-PrOH $(2 \mathrm{~mL})$ was stirred at room temperature. The reaction mixture was stirred for $5 \mathrm{~h}$. The mixture was filtered off, washed with diethyl ether and dried in a vacuum to give a product as a white solid (231 mg, 82\%): m.p: $207 \square ;{ }^{1} \mathrm{H}$ NMR (500 MHz, D $\left.2 \mathrm{O}\right) \delta 4.71(\mathrm{t}, J=9.0 \mathrm{~Hz}, 1 \mathrm{H}), 4.32$ (dd, $J=8.0$ and $12.0 \mathrm{~Hz}, 1 \mathrm{H}), 3.68(\mathrm{dd}, J=8.5$ and $12 \mathrm{~Hz}, 1 \mathrm{H}), 3.21(\mathrm{~s}, 3 \mathrm{H}), 3.15$ (s, $3 \mathrm{H}), 3.10$ (s, 3H); ${ }^{13} \mathrm{C}$ NMR (125 MHz, $\left.\mathrm{D}_{2} \mathrm{O}\right)$ 176.60, 68.70, 58.20, 57.24, 55.60, 40.75; IR (Ge ATR) 3331, 3137, 1616, 1400, 1367, 1311, $1154 \mathrm{~cm}^{-1}$. Anal. Calcd for 
Supporting Information: Efficient Synthesis of Highly Functionalized Cyclic Aminimides; Moon, Han, and Kim May 31, 2005.

$\mathrm{C}_{6} \mathrm{H}_{13} \mathrm{~N}_{3} \mathrm{O}_{3} \mathrm{~S}$ : C, 34.77; H, 6.32; Found: C, 34.13; H, 6.14. LRMS (ESI): $m / z 229.8$ (230.1 calcd for $\mathrm{C}_{6} \mathrm{H}_{13} \mathrm{~N}_{3} \mathrm{O}_{3} \mathrm{SNa}[\mathrm{M}+\mathrm{Na}]^{+}$).

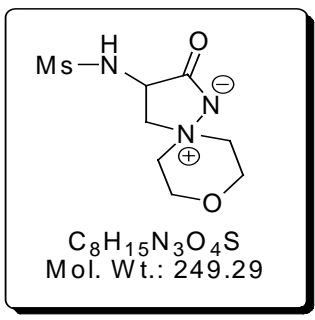

$N$-(2-oxo-8-oxa-1,5-diaza-spiro[4,5]dec-3-yl)-methanesulfoamide (29): To a solution of 1-methanesulfonyl-aziridine-2-carboxylic acid methyl ester (500 mg, $2.79 \mathrm{mmol}$ ), $\mathrm{N}$ aminomorphorine $(323 \mu \mathrm{L}, 1.06 \mathrm{mmol})$ in $i-\mathrm{PrOH}(3 \mathrm{~mL})$ was stirred at room temperature for $6 \mathrm{~h}$. The mixture was filtered off, washed with diethyl ether and dried in a vacuum to give a product as a white solid (536 mg, 77\%): m.p: $219 \square$; ${ }^{1} \mathrm{H}$ NMR (500 $\left.\mathrm{MHz}, \mathrm{D}_{2} \mathrm{O}\right) \delta 4.67(\mathrm{t}, J=7.5 \mathrm{~Hz}, 1 \mathrm{H}), 4.39(\mathrm{dd}, J=8.5$ and $12.5 \mathrm{~Hz}, 1 \mathrm{H}), 4.07(\mathrm{~m}, 2 \mathrm{H})$, $3.90(\mathrm{~m}, 2 \mathrm{H}), 3.69(\mathrm{dd}, J=8.5$ and $12.0 \mathrm{~Hz}, 1 \mathrm{H}), 3.56(\mathrm{~m}, 2 \mathrm{H}), 3.38(\mathrm{~m}, 2 \mathrm{H}), 3.10$ (s, $3 \mathrm{H}) ;{ }^{13} \mathrm{C}$ NMR (125 MHz, $\left.\mathrm{D}_{2} \mathrm{O}\right)$ 176.94, 67.44, 65.39, 64.59, 62.32, 62.24, 54.31, 40.77; Anal. Calcd for $\mathrm{C}_{8} \mathrm{H}_{15} \mathrm{~N}_{3} \mathrm{O}_{4} \mathrm{~S}$ : C, 38.54; H, 6.06; N, 16.86. Found: C, 38.97; H, $5.81 ; \mathrm{N}, 16.46$. 
Supporting Information: Efficient Synthesis of Highly Functionalized Cyclic Aminimides; Moon, Han, and Kim May 31, 2005.

\section{Crystallographic Data for 14}

\section{Data collection (14)}

A dark orange crystal with approximate dimensions $0.30 \times 0.22 \times 0.10 \mathrm{~mm}^{3}$ was selected and attached to the tip of a glass fiber, transferred to a Bruker SMART diffractometer/CCD area detector and centered under liquid nitrogen in the beam at 233(2)K.

The crystal evaluation and data collection were performed on a Bruker CCD diffractometer with $\mathrm{Mo} \mathrm{K}_{\alpha}(\lambda=0.71073)$ radiation employing $2 \mathrm{kw}$ sealed tube X-ray source operating at $1.6 \mathrm{~kW}$ and the diffractometer to crystal distance of $4.9701 \mathrm{~cm}$.

Preliminary orientation matrix and cell constants were determined from three series of $\omega$ scans at different starting angles. Each series consisted of 10 frames collected at intervals of $0.3^{\circ} \omega$ scan with the exposure time of 10 seconds per frame. The reflections were successfully indexed by an automated indexing routine built in the SMART program. ${ }^{1}$

A total of 4681 data were harvested by collecting three sets of frames with $0.3^{\circ} \mathrm{\omega}$ scans with an exposure time 10 seconds per frame. The highly redundant datasets were corrected for Lorentz and polarization effects, but no correction for crystal decay was applied.

\section{$\underline{\text { Structure Solution and Refinement }}$}

The systematic absences in the diffraction data were consistent for the space group, Monoclinic, $\mathrm{P} 2{ }_{1} / \mathrm{c}$ that yielded chemically reasonable ${ }^{3}$ and computationally stable results of refinement. ${ }^{4}$

A successful solution was obtained by the direct methods ${ }^{5}$ from the $E$-map. The remaining non-hydrogen atoms were located in an alternating series of least-squares cycles and difference Fourier maps. All non-hydrogen atoms were refined with anisotropic displacement coefficients. All hydrogen atoms were included in the structure factor calculation at idealized positions and were allowed to ride on the neighboring atoms with relative isotropic displacement coefficients. The final least-squares refinement of 109 parameters against 1895 data resulted in $R_{1}$ (based on $F_{o}$ for $F_{o}>4 \sigma$ ) and $\mathrm{wR}_{2}$ (based on $F^{2}$ for all data) of 0.0799 and 0.1774 , respectively.

\section{$\underline{\text { References }}$}


Supporting Information: Efficient Synthesis of Highly Functionalized Cyclic Aminimides; Moon, Han, and Kim May 31, 2005.

1. SMART Area-Detector Software Package, Bruker AXS, Inc., Madison WI, 1995.

2. SAINT; SAX Area-Detector Integration Program, version 4.050, Bruker AXS, Inc., Madison WI, 1995.

3. XPREP: part of the SHELXTL, Crystal Structure Determination Package, version 5.04, Bruker AXS, Inc., Madison WI, 1995.

4. SHELX-97 Sheldrick, G. M. Institut fur Anorganische chemie der Universitat, Gotingen, F. R. G., 1997.

5. Beurskens, P. T. Admiral G. Beuskens, G. Bosman, W. P. Garcia-Granda, S. Gould, R. O. Smits, J. M. M. and Smkalla, C. DIRIF 92. The DIRIF Pragram System, Technical Report of the Crystallography Laboratory, University of Nijmegen, 1992. 

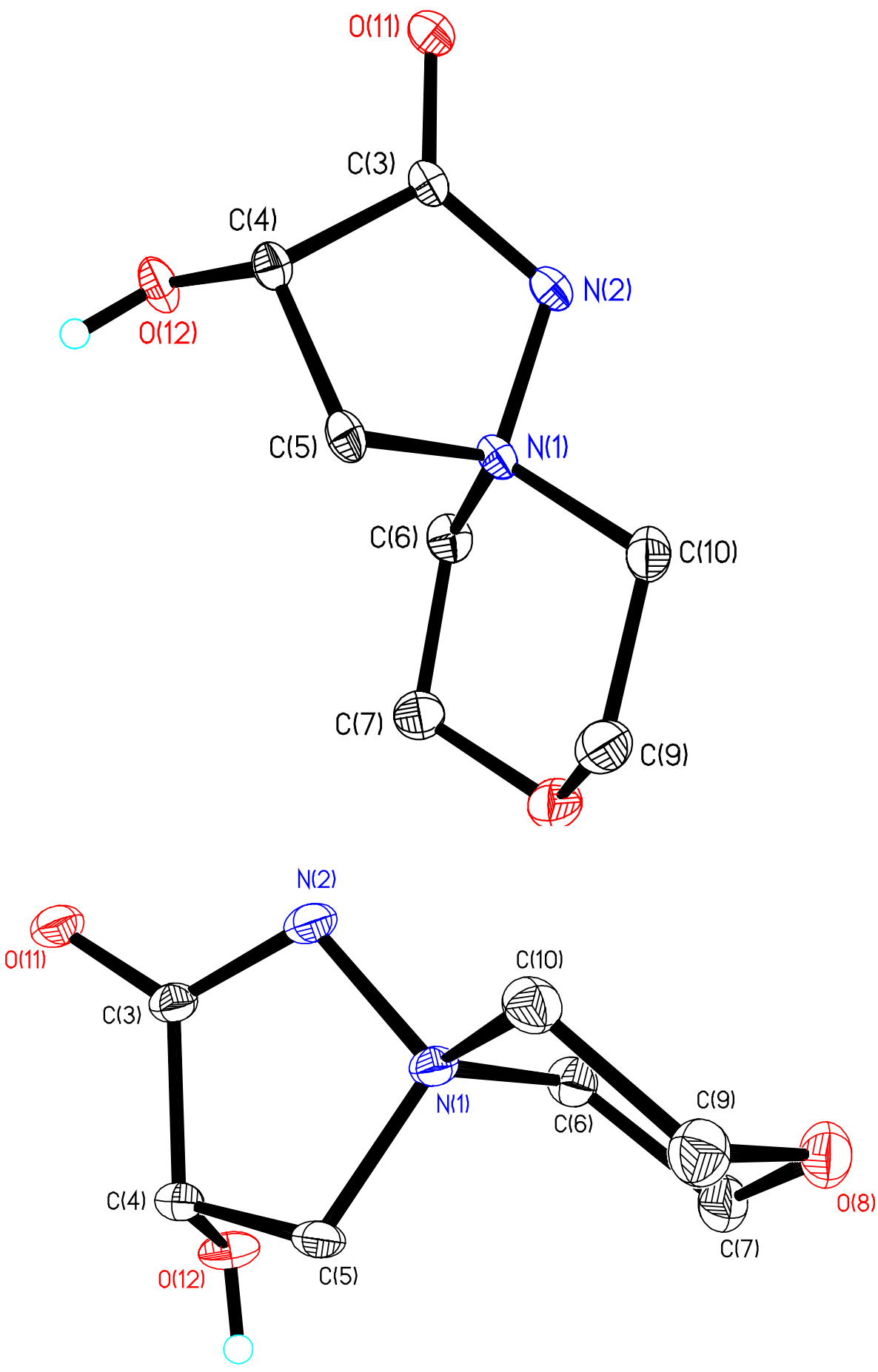

Ortep drawing of G012 : for clarity, hydrogens were omitted 
Supporting Information: Efficient Synthesis of Highly Functionalized Cyclic Aminimides; Moon, Han, and Kim May 31, 2005.

Table 1. Crystal data and structure refinement for $\mathbf{1 4 .}$

Identification code

Empirical formula

Formula weight

Temperature

Wavelength

Crystal system, space group

Unit cell dimensions

Volume

Z, Calculated density

Absorption coefficient

$\mathrm{F}(000)$

Crystal size

Theta range for data collection

Limiting indices

Reflections collected / unique

Completeness to theta $=28.03$

Max. and min. transmission

Refinement method

Data / restraints / parameters

Goodness-of-fit on $\mathrm{F}^{2}$

Final $R$ indices [I $>2 \operatorname{sigma}(\mathrm{I})]$

$\mathrm{R}$ indices (all data)

Largest diff. peak and hole
14

$\mathrm{C}_{7} \mathrm{H}_{12} \mathrm{~N}_{2} \mathrm{O}_{3}$

172.19

233(2) K

$0.71073 \AA$

Monoclinic, P2(1)/c

$\mathrm{a}=5.520(3) \AA \quad$ alpha $=90 \mathrm{deg}$.

$\mathrm{b}=8.698(4) \AA \quad$ beta $=98.120(7) \mathrm{deg}$.

$\mathrm{c}=16.224(7) \AA \quad$ gamma $=90 \mathrm{deg}$.

771.1(6) $\AA^{3}$

4, $\quad 1.483 \mathrm{Mg} / \mathrm{m}^{3}$

$0.117 \mathrm{~mm}^{-1}$

368

$0.30 \times 0.22 \times 0.10 \mathrm{~mm}$

2.54 to $28.03 \mathrm{deg}$.

$-7<=\mathrm{h}<=7,-7<=\mathrm{k}<=11,-21<=\mathrm{l}<=19$

$4681 / 1859[\mathrm{R}(\mathrm{int})=0.0735]$

$98.9 \%$

0.9884 and 0.9659

Full-matrix least-squares on $\mathrm{F}^{2}$

1859 / 0 / 109

1.066

$\mathrm{R}_{1}=0.0799, \mathrm{wR}_{2}=0.1549$

$\mathrm{R}_{1}=0.1351, \mathrm{wR}_{2}=0.1774$

0.294 and -0.236 e. $\AA^{-3}$ 
Supporting Information: Efficient Synthesis of Highly Functionalized Cyclic Aminimides; Moon, Han, and Kim May 31, 2005.

Table 2. Atomic coordinates ( $\times 10^{4}$ ) and equivalent isotropic displacement parameters $\left(\AA^{2} \times 10^{3}\right)$ for 14 . U(eq) is defined as one third of the trace of the orthogonalized Uij tensor.

\begin{tabular}{llrrr}
\hline & $\mathrm{x}$ & $\mathrm{y}$ & $\mathrm{z}$ & $\mathrm{U}(\mathrm{eq})$ \\
\hline $\mathrm{O}(11)$ & $3447(3)$ & $1495(2)$ & $3576(1)$ & $29(1)$ \\
$\mathrm{O}(12)$ & $8683(3)$ & $732(2)$ & $3412(1)$ & $30(1)$ \\
$\mathrm{N}(1)$ & $5844(4)$ & $1863(2)$ & $1716(1)$ & $20(1)$ \\
$\mathrm{N}(2)$ & $3799(4)$ & $1609(3)$ & $2193(1)$ & $25(1)$ \\
$\mathrm{O}(8)$ & $7517(4)$ & $1424(2)$ & $124(1)$ & $33(1)$ \\
$\mathrm{C}(3)$ & $4678(5)$ & $1710(3)$ & $2992(2)$ & $21(1)$ \\
$\mathrm{C}(6)$ & $6618(5)$ & $318(3)$ & $1425(2)$ & $25(1)$ \\
$\mathrm{C}(5)$ & $7834(5)$ & $2661(3)$ & $2303(2)$ & $22(1)$ \\
$\mathrm{C}(10)$ & $4947(5)$ & $2800(3)$ & $961(2)$ & $27(1)$ \\
$\mathrm{C}(7)$ & $8435(6)$ & $488(4)$ & $816(2)$ & $31(1)$ \\
$\mathrm{C}(4)$ & $7410(5)$ & $2098(3)$ & $3146(2)$ & $21(1)$ \\
$\mathrm{C}(9)$ & $6894(6)$ & $2910(4)$ & $395(2)$ & $33(1)$ \\
\hline
\end{tabular}


Supporting Information: Efficient Synthesis of Highly Functionalized Cyclic Aminimides; Moon, Han, and Kim May 31, 2005.

Table 3. Bond lengths $[\AA]$ and angles $[\mathrm{deg}]$ for 14.

\begin{tabular}{lc}
\hline $\mathrm{O}(11)-\mathrm{C}(3)$ & $1.256(3)$ \\
$\mathrm{O}(12)-\mathrm{C}(4)$ & $1.416(3)$ \\
$\mathrm{N}(1)-\mathrm{N}(2)$ & $1.473(3)$ \\
$\mathrm{N}(1)-\mathrm{C}(10)$ & $1.495(3)$ \\
$\mathrm{N}(1)-\mathrm{C}(6)$ & $1.506(3)$ \\
$\mathrm{N}(1)-\mathrm{C}(5)$ & $1.516(3)$ \\
$\mathrm{N}(2)-\mathrm{C}(3)$ & $1.322(3)$ \\
$\mathrm{O}(8)-\mathrm{C}(7)$ & $1.421(4)$ \\
$\mathrm{O}(8)-\mathrm{C}(9)$ & $1.423(4)$ \\
$\mathrm{C}(3)-\mathrm{C}(4)$ & $1.531(4)$ \\
$\mathrm{C}(6)-\mathrm{C}(7)$ & $1.511(4)$ \\
$\mathrm{C}(5)-\mathrm{C}(4)$ & $1.503(4)$ \\
$\mathrm{C}(10)-\mathrm{C}(9)$ & $1.512(4)$ \\
$\mathrm{N}(2)-\mathrm{N}(1)-\mathrm{C}(10)$ & $108.8(2)$ \\
$\mathrm{N}(2)-\mathrm{N}(1)-\mathrm{C}(6)$ & $107.65(19)$ \\
$\mathrm{C}(10)-\mathrm{N}(1)-\mathrm{C}(6)$ & $107.8(2)$ \\
$\mathrm{N}(2)-\mathrm{N}(1)-\mathrm{C}(5)$ & $106.24(19)$ \\
$\mathrm{C}(10)-\mathrm{N}(1)-\mathrm{C}(5)$ & $113.0(2)$ \\
$\mathrm{C}(6)-\mathrm{N}(1)-\mathrm{C}(5)$ & $113.1(2)$ \\
$\mathrm{C}(3)-\mathrm{N}(2)-\mathrm{N}(1)$ & $107.6(2)$ \\
$\mathrm{C}(7)-\mathrm{O}(8)-\mathrm{C}(9)$ & $110.6(2)$ \\
$\mathrm{O}(11)-\mathrm{C}(3)-\mathrm{N}(2)$ & $124.6(3)$ \\
$\mathrm{O}(11)-\mathrm{C}(3)-\mathrm{C}(4)$ & $122.3(2)$ \\
$\mathrm{N}(2)-\mathrm{C}(3)-\mathrm{C}(4)$ & $113.1(2)$ \\
$\mathrm{N}(1)-\mathrm{C}(6)-\mathrm{C}(7)$ & $111.2(2)$ \\
$\mathrm{C}(4)-\mathrm{C}(5)-\mathrm{N}(1)$ & $103.3(2)$ \\
$\mathrm{N}(1)-\mathrm{C}(10)-\mathrm{C}(9)$ & $110.5(2)$ \\
$\mathrm{O}(8)-\mathrm{C}(7)-\mathrm{C}(6)$ & $112.1(2)$ \\
$\mathrm{O}(12)-\mathrm{C}(4)-\mathrm{C}(5)$ & $114.6(2)$ \\
$\mathrm{O}(12)-\mathrm{C}(4)-\mathrm{C}(3)$ & \\
$\mathrm{C}(5)-\mathrm{C}(4)-\mathrm{C}(3)$ & $\mathrm{O}(8)-\mathrm{C}(9)-\mathrm{C}(10)$ \\
& \\
& \\
& $107(2)$ \\
& \\
\hline
\end{tabular}

Symmetry transformations used to generate equivalent atoms: 
Supporting Information: Efficient Synthesis of Highly Functionalized Cyclic Aminimides; Moon, Han, and Kim May 31, 2005.

Table 4. Anisotropic displacement parameters $\left(\AA^{2} \times 10^{3}\right)$ for 14. The anisotropic displacement factor exponent takes the form: $-2 \mathrm{pi}^{2}\left[\mathrm{~h}^{2} \mathrm{a}^{*^{2}} \mathrm{U} 11+\ldots+2 \mathrm{~h} \mathrm{k} \mathrm{a}^{*} \mathrm{~b}^{*}\right.$ U12 ]

\begin{tabular}{llllrrr}
\hline & $\mathrm{U} 11$ & $\mathrm{U} 22$ & $\mathrm{U} 33$ & $\mathrm{U} 23$ & $\mathrm{U} 13$ & $\mathrm{U} 12$ \\
\hline $\mathrm{O}(11)$ & $19(1)$ & $41(1)$ & $28(1)$ & $1(1)$ & $6(1)$ & $-2(1)$ \\
$\mathrm{O}(12)$ & $15(1)$ & $34(1)$ & $40(1)$ & $8(1)$ & $1(1)$ & $1(1)$ \\
$\mathrm{N}(1)$ & $14(1)$ & $22(1)$ & $25(1)$ & $-2(1)$ & $3(1)$ & $1(1)$ \\
$\mathrm{N}(2)$ & $15(1)$ & $33(1)$ & $27(1)$ & $1(1)$ & $5(1)$ & $0(1)$ \\
$\mathrm{O}(8)$ & $36(1)$ & $38(1)$ & $26(1)$ & $-4(1)$ & $6(1)$ & $4(1)$ \\
$\mathrm{C}(3)$ & $15(1)$ & $21(2)$ & $26(2)$ & $0(1)$ & $2(1)$ & $1(1)$ \\
$\mathrm{C}(6)$ & $26(2)$ & $19(2)$ & $29(2)$ & $-3(1)$ & $3(1)$ & $2(1)$ \\
$\mathrm{C}(5)$ & $14(1)$ & $23(2)$ & $28(2)$ & $-3(1)$ & $0(1)$ & $-3(1)$ \\
$\mathrm{C}(10)$ & $25(2)$ & $29(2)$ & $27(2)$ & $0(1)$ & $-1(1)$ & $4(1)$ \\
$\mathrm{C}(7)$ & $32(2)$ & $35(2)$ & $28(2)$ & $-6(1)$ & $5(1)$ & $3(1)$ \\
$\mathrm{C}(4)$ & $16(1)$ & $23(2)$ & $25(1)$ & $0(1)$ & $1(1)$ & $-2(1)$ \\
$\mathrm{C}(9)$ & $35(2)$ & $35(2)$ & $28(2)$ & $2(1)$ & $5(1)$ & $-1(1)$ \\
\hline
\end{tabular}


Supporting Information: Efficient Synthesis of Highly Functionalized Cyclic Aminimides; Moon, Han, and Kim May 31, 2005.

Table 5. Hydrogen coordinates $\left(\mathrm{x} 10^{4}\right)$ and isotropic displacement parameters $\left(\AA^{2} \mathrm{x}\right.$ $10^{3}$ ) for 14.

\begin{tabular}{lrrrc}
\hline & \multicolumn{2}{l}{$\mathrm{y}$} & $\mathrm{z}$ & $\mathrm{U}(\mathrm{eq})$ \\
\hline $\mathrm{H}(12 \mathrm{~A})$ & 10161 & 895 & 3472 & 45 \\
$\mathrm{H}(6 \mathrm{~A})$ & 5190 & -234 & 1160 & 30 \\
$\mathrm{H}(6 \mathrm{~B})$ & 7352 & -277 & 1901 & 30 \\
$\mathrm{H}(5 \mathrm{~A})$ & 9446 & 2369 & 2185 & 26 \\
$\mathrm{H}(5 \mathrm{~B})$ & 7670 & 3770 & 2263 & 26 \\
$\mathrm{H}(10 \mathrm{~A})$ & 4522 & 3824 & 1130 & 33 \\
$\mathrm{H}(10 \mathrm{~B})$ & 3488 & 2330 & 663 & 33 \\
$\mathrm{H}(7 \mathrm{~A})$ & 9932 & 937 & 1099 & 37 \\
$\mathrm{H}(7 \mathrm{~B})$ & 8827 & -522 & 617 & 37 \\
$\mathrm{H}(4 \mathrm{~A})$ & 7745 & 2911 & 3565 & 25 \\
$\mathrm{H}(9 \mathrm{~A})$ & 6300 & 3540 & -85 & 39 \\
$\mathrm{H}(9 \mathrm{~B})$ & 8341 & 3400 & 691 & 39 \\
& & & & \\
\hline
\end{tabular}


Supporting Information: Efficient Synthesis of Highly Functionalized Cyclic Aminimides; Moon, Han, and Kim May 31, 2005.

Table 6. Torsion angles [deg] for 14.

\begin{tabular}{lr}
\hline $\mathrm{C}(10)-\mathrm{N}(1)-\mathrm{N}(2)-\mathrm{C}(3)$ & $-141.1(2)$ \\
$\mathrm{C}(6)-\mathrm{N}(1)-\mathrm{N}(2)-\mathrm{C}(3)$ & $102.3(2)$ \\
$\mathrm{C}(5)-\mathrm{N}(1)-\mathrm{N}(2)-\mathrm{C}(3)$ & $-19.1(3)$ \\
$\mathrm{N}(1)-\mathrm{N}(2)-\mathrm{C}(3)-\mathrm{O}(11)$ & $-177.2(2)$ \\
$\mathrm{N}(1)-\mathrm{N}(2)-\mathrm{C}(3)-\mathrm{C}(4)$ & $1.9(3)$ \\
$\mathrm{N}(2)-\mathrm{N}(1)-\mathrm{C}(6)-\mathrm{C}(7)$ & $170.9(2)$ \\
$\mathrm{C}(10)-\mathrm{N}(1)-\mathrm{C}(6)-\mathrm{C}(7)$ & $53.7(3)$ \\
$\mathrm{C}(5)-\mathrm{N}(1)-\mathrm{C}(6)-\mathrm{C}(7)$ & $-72.0(3)$ \\
$\mathrm{N}(2)-\mathrm{N}(1)-\mathrm{C}(5)-\mathrm{C}(4)$ & $28.4(3)$ \\
$\mathrm{C}(10)-\mathrm{N}(1)-\mathrm{C}(5)-\mathrm{C}(4)$ & $147.7(2)$ \\
$\mathrm{C}(6)-\mathrm{N}(1)-\mathrm{C}(5)-\mathrm{C}(4)$ & $-89.5(2)$ \\
$\mathrm{N}(2)-\mathrm{N}(1)-\mathrm{C}(10)-\mathrm{C}(9)$ & $-172.5(2)$ \\
$\mathrm{C}(6)-\mathrm{N}(1)-\mathrm{C}(10)-\mathrm{C}(9)$ & $-56.0(3)$ \\
$\mathrm{C}(5)-\mathrm{N}(1)-\mathrm{C}(10)-\mathrm{C}(9)$ & $69.8(3)$ \\
$\mathrm{C}(9)-\mathrm{O}(8)-\mathrm{C}(7)-\mathrm{C}(6)$ & $58.2(3)$ \\
$\mathrm{N}(1)-\mathrm{C}(6)-\mathrm{C}(7)-\mathrm{O}(8)$ & $-55.8(3)$ \\
$\mathrm{N}(1)-\mathrm{C}(5)-\mathrm{C}(4)-\mathrm{O}(12)$ & $89.7(3)$ \\
$\mathrm{N}(1)-\mathrm{C}(5)-\mathrm{C}(4)-\mathrm{C}(3)$ & $-25.7(3)$ \\
$\mathrm{O}(11)-\mathrm{C}(3)-\mathrm{C}(4)-\mathrm{O}(12)$ & $74.4(3)$ \\
$\mathrm{N}(2)-\mathrm{C}(3)-\mathrm{C}(4)-\mathrm{O}(12)$ & $-104.7(3)$ \\
$\mathrm{O}(11)-\mathrm{C}(3)-\mathrm{C}(4)-\mathrm{C}(5)$ & $-165.0(2)$ \\
$\mathrm{N}(2)-\mathrm{C}(3)-\mathrm{C}(4)-\mathrm{C}(5)$ & $15.9(3)$ \\
$\mathrm{C}(7)-\mathrm{O}(8)-\mathrm{C}(9)-\mathrm{C}(10)$ & \\
$\mathrm{N}(1)-\mathrm{C}(10)-\mathrm{C}(9)-\mathrm{O}(8)$ & \\
& \\
& \\
& \\
& \\
&
\end{tabular}

Symmetry transformations used to generate equivalent atoms: 